\title{
Answer to the Letter to the Editor of A.T. Turgut et al. concerning "Intradural extramedullary primary hydatid cyst of the spine in a child: a very rare presentation"
}

\author{
S. H. Arif · Sufian Zaheer
}

Accepted: 8 April 2009/Published online: 24 April 2009

(C) Springer-Verlag 2009

\section{Respected Sir,}

Thanks for such an in-depth critical re-review of our article, we highly appreciate it.

As a part of routine investigation USG abdomen was done and no cyst was found in any other part of the abdomen, and in primary MRI itself no cystic lesion was present in any other part of the body otherwise we must have mentioned it in our case report. In fact, as mentioned in our case report, in post-operative MRI (after 6 months) there was no cystic lesion present in any part of the body. Unfortunately the CT scan of the patient was not available.

The details of MRI were provided by the radiologists and they mentioned it to be the cystic lesion extending from the L1 to L4 lumbar spine.

The staining was done with the hematoxylin \& eosin, and the ratio of magnification for figure 3 is $\times 400$ and that for figure 4 is $\times 1,000$.

As for not inclusion of the names of radiologists and spinal surgeon, we wanted to say that we had tried to discuss the case from the pathologists point of view and wanted to give the message to radiologists and the surgeons that although the hydatid cyst is rare in spine, the possibility should always be kept in mind especially in endemic areas, which was also not thought of in this particular case, and the diagnosis of arachnoid cyst was made by the radiologist, and the surgeon operated the patient keeping in mind the arachnoid cyst, and it was only when the material submitted for histopathological examination that the diagnosis of hydatid cyst was made.

Post operatively the patient received albedazole $800 \mathrm{mg}$ daily in two divided doses for 6 months. The weakness of the lower limb at the first neurological examination at the admission to the hospital was Grade 3. We totally agree that long-term follow-up is mandatory in each case of central nervous hydatidosis before any conclusion can be drawn about the value of any therapeutic agent.
S. H. Arif · S. Zaheer $(\bowtie)$

Jawaharlal Nehru Medical College, Aligarh Muslim University,

Aligarh 202002, Uttar Pradesh, India

e-mail: sufianzaheer@gmail.com 